\title{
Severe Acute Asthma: An Attempt to Identify the Quiet Before the Storm
}

Asthma is a serious and prevalent public health problem that spans the entire world. It is estimated that asthma affects over 300 million people worldwide. ${ }^{1}$ Its prevalence is approximately 21 million citizens in the United States, according to the Centers for Disease Control and Prevention. ${ }^{2}$ Other reports indicate that as many as 9.5 million children currently have asthma in the United States. ${ }^{3}$ Asthma care and management have undergone much scrutiny over the last 30 years, which has led to the development and implementation of guidelines throughout the world that have recently gravitated toward being evidencebased. Evidence-based clinical practice guidelines offer both evidence and instructional components, and represent the ideal for bringing current scientific knowledge to the clinician and bedside. ${ }^{4}$

The topic of fatal or near fatal or severe acute asthma has intrigued the respiratory, pulmonary, and epidemiology communities over several decades. The treatment and management of severe acute asthma is often the least scientifically supported subject documented in asthma guidelines. In fact, a precise definition of a severe asthmatic exacerbation is an issue that presents difficulties. ${ }^{5}$ The term "status asthmaticus" relates severity to outcome and has been used to define a severe asthmatic exacerbation that does not respond to and/or perilously delays the repetitive or continuous administration of short-acting inhaled $\beta_{2}$ adrenergic receptor agonists in the emergency setting. ${ }^{6}$

Despite this lack of clarity of what constitutes severe acute asthma, it occurs across the entire disease severity spectrum in pediatrics. In a study by Fuhlbrigge and colleagues, ${ }^{7}$ they explored the relationship between percentpredicted $\mathrm{FEV}_{1}\left(\mathrm{FEV}_{1} \%\right)$ and subsequent asthma attacks in a longitudinal study of pediatric lung health. This study documented that, regardless of $\mathrm{FEV}_{1} \%$, approximately 30 $65 \%$ of children with asthma reported an exacerbation over the past year.

Most asthma guidelines offer evidence-based or best practice approaches to the management of asthma exacerbations. ${ }^{4}$ Most guidelines struggle with the presentation of a robust evidence-based approach for life-threatening or severe asthma exacerbations. Despite the lack of evidence, much ongoing scientific interest exists and is written about when it comes to life-threatening asthma or severe asthma exacerbations. ${ }^{8}$ The rationale, of course, is that if the pathophysiology or phenotype of children who suffer severe asthma attacks could be better understood, the interventions and treatments provided could be instituted much sooner, leading to quicker resolution of the exacerbation, with less functional morbidity.

See the Original Study on Page 1391

In this edition of Respiratory CARE, van den Bosch and colleagues ${ }^{9}$ describe a multicenter retrospective casecontrolled study in the Netherlands, which they undertook in an attempt to identify risk factors for pediatric ICU (PICU) admissions. One of the inherent problems with retrospective studies is they are often difficult to generalize to other populations, as they typically do not utilize control groups and frequently encounter difficulty with obtained results, as correlation does not imply causation.

The authors attempted to overcome inherent biases of retrospective studies by focusing on a known pediatric asthma population, by limiting their recruitment criteria to children diagnosed with asthma and being treated by pediatricians or pediatric pulmonologists in out-patient clinics from 3 general hospitals and one university hospital. To provide a control group for comparison they compared the group of children admitted to the PICU to similar patients from the same participating referring hospitals and physician management that never needed PICU admission for any reason.

Van den Bosch and colleagues ${ }^{9}$ then identified from previous studies several risk factors that had been identified for severe asthma attacks that led to PICU admissions. The risk factors were categorized into 4 main domains: patient characteristics, ethnicity and psychosocial context, exposures and atopy, and severity and treatment of asthma. These data were extracted from the medical records of the children's pediatrician or pulmonologist a few months prior to the emergency room visit. As with any retrospective study, this area was somewhat problematic in this paper, as not all patients had completed records that identified the 25 variables that were to be assessed, with the threshold set at $30 \%$ of data necessary for inclusion. The challenge is that the missing risk variables may or may not have 


\section{Severe Acute Asthma: An Attempt to Identify the Quiet Before the Storm}

altered the overall final analysis for PICU admission risk factors.

In the univariate analysis, all risk characteristics studied, with the exception of prescribed corticosteroids, demonstrated significant difference for those admitted to the PICU versus those not admitted. So each as a risk factor in theory provided an increased risk for PICU admission, compared to the match controls, which stands to reason, as theses variables were selected based on their increased risk from previous studies. The characteristics of pets $(38 \%)$, smoking (31\%), sanitized homes (48\%), hay fever (41\%), and severe asthma (54\%) were all missing a sizeable portion of the 2 study groups' data sets. This leads to the question, would the missing data have made a difference in any of these variables achieving statistical significance had it been available?

The authors also conducted a multivariate analysis to determine if multiple characteristics combined placed children with severe acute asthma at greater risk for PICU admission than their control counterparts. This multivariate analysis showed that only (active or passive) smoking, allergies, earlier hospitalization for asthma, and nonsanitized home proved to be significant independent risk factors for PICU admission. While these variables may not be surprising to those who work in pediatric institutions or with children with asthma, the concern for all should be that these are all very common triggers (allergies, smoke exposure, and environmental triggers in the home) or events (hospitalizations for asthma).

The study by van den Bosch is an important start in "real world" studies to identify children who are at risk for severe asthma attacks and who do not respond to traditional asthma therapies ( $\beta$ agonists and corticosteroids) and require a higher level of care and treatment in a PICU setting. A recent study by $\mathrm{Wu}$ and colleagues ${ }^{10}$ suggested that a child experiencing persistent asthma symptoms is closely associated with having severe exacerbations from asthma with predictors of treatment with inhaled corticosteroids, lower $\mathrm{PC}_{20}$ (provocational concentration that produces a $20 \%$ decrease in $\mathrm{FEV}_{1}$ ), and lower $\mathrm{FEV}_{1} / \mathrm{FVC}$. An older review by Werner indicated that prior PICU admission due to severe acute asthma has been shown to be a risk factor for readmission because of severe acute asthma. ${ }^{11}$

The author has disclosed no conflicts of interest.

Correspondence: Timothy R Myers RRT-NPS, Pediatric Diagnostics and Respiratory Care, Rainbow Babies and Children's Hospital, 11100 Euclid Avenue, Mailstop 6043, Cleveland OH 44106. E-mail: timothy.myers@uhhospitals.org.

DOI: $10.4187 /$ respcare. 02070
Avoidance of ICU admission and the risk of more invasive interventions are highly desirable goals of pediatric asthma management, the rationale being that children admitted to the ICU and intubated are more at risk for comorbidities or a prolonged recovery period, with substantial functional morbidities. In fact, a relatively high proportion (17 of 139 or $12 \%$ ) of the patients in this retrospective analysis ${ }^{9}$ required intubation and mechanical ventilation, with 3 developing air leak.

Studies that are focused on identifying patients at risk for asthma exacerbations are a key component of asthma guidelines. Identifying characteristics of increased risk enables clinicians and researchers to develop interventions and education to minimize these risks or at least to provide concentrated education to patients and their caregivers.

Timothy R Myers MBA RRT-NPS

Pediatric Diagnostics and Respiratory Care Rainbow Babies and Children's Hospital Case Western Reserve University School of Medicine Cleveland, Ohio

\section{REFERENCES}

1. Global Initiative for Asthma (GINA). Global strategy for asthma management and prevention, updated December 2011. http://www. ginasthma.org/guidelines-gina-report-global-strategy-for-asthma. html. Accessed June 25, 2012.

2. Moorman JE, Rudd RA, Johnson CA, King M, Minor P, Bailey C, et al. National surveillance for asthma: United States, 1980-2004. MMWR Surveill Summ 2007;56(8):1-54.

3. Myers TR, Tomasio L. Asthma 2015 and beyond. Respir Care 2011; 56(9):1389-1407.

4. Myers TR. Guidelines for asthma management: a review and comparison of 5 current guidelines. Respir Care 2008;53(6):751-767.

5. McFadden ER Jr. Acute severe asthma. Am J Respir Crit Care Med 2003;168:740-59.

6. Papiris SA, Manali ED, Kolilekas L, Triantafillidou C, Tsangaris I. Acute severe asthma: new approaches to assessment and treatment. Drugs 2009;69(17):2363-2391.

7. Fuhlbrigge AL, Kitch BT, Paltiel AD, Kuntz KM, Neumann PJ, Dockery DW, Weiss ST. FEV 1 is associated with risk of asthma attacks in a pediatric population. J Allergy Clin Immunol 2001; 107(1):61-67.

8. Rubin BK, Pohank V. Beyond the guidelines: fatal and near-fatal asthma. Paediatric Respir Rev 2012;13(2):106-111.

9. van den Bosch GE, Merkus PJFM, Buysse CMP, Boehmer ALM, Vaessen-Verberne AAPH, van Veen LN, Hop WCJ, de Hoog M. Risk factors for pediatric intensive care admission in children with acute asthma. Respir Care 2012;57(9):1391-1397.

10. Wu AC, Tantisira K, Li L, Schuemann B, Weiss ST, Fuhlbrigge AL; Childhood Asthma Management Program Research Group. Predictors of symptoms are different from predictors of severe exacerbations from asthma in children. Chest 2011;140(1):100-107.

11. Werner HA. Status asthmaticus in children: a review. Chest 2001; 119(6):1913-1929. 